\title{
Hot Flow Testing of a Film Cooled Dual Bell Nozzle
}

\author{
Ralf H. Stark ${ }^{1}$ and Chloé Génin ${ }^{2}$ \\ German Aerospace Center, Lampoldshausen, Germany, D-74239
}

\begin{abstract}
The altitude adaptive dual bell nozzles can increase the overall performance of existing main stage rocket engines like Europe's Vulcain 2. The Institute of Space propulsion of the German Aerospace Center in Lampoldshausen carried out in the past various cold and warm flow subscale tests to study the flow physics of dual bell nozzles fundamentally. Following this line and in order to increase the technology readiness level hot flow subscale tests were conducted to study the effects of a cooling film on the characteristics of a dual bell nozzle. The experimental results of a first campaign, performed with a representative Vulcain 2 like subscale engine, are presented and discussed.
\end{abstract}

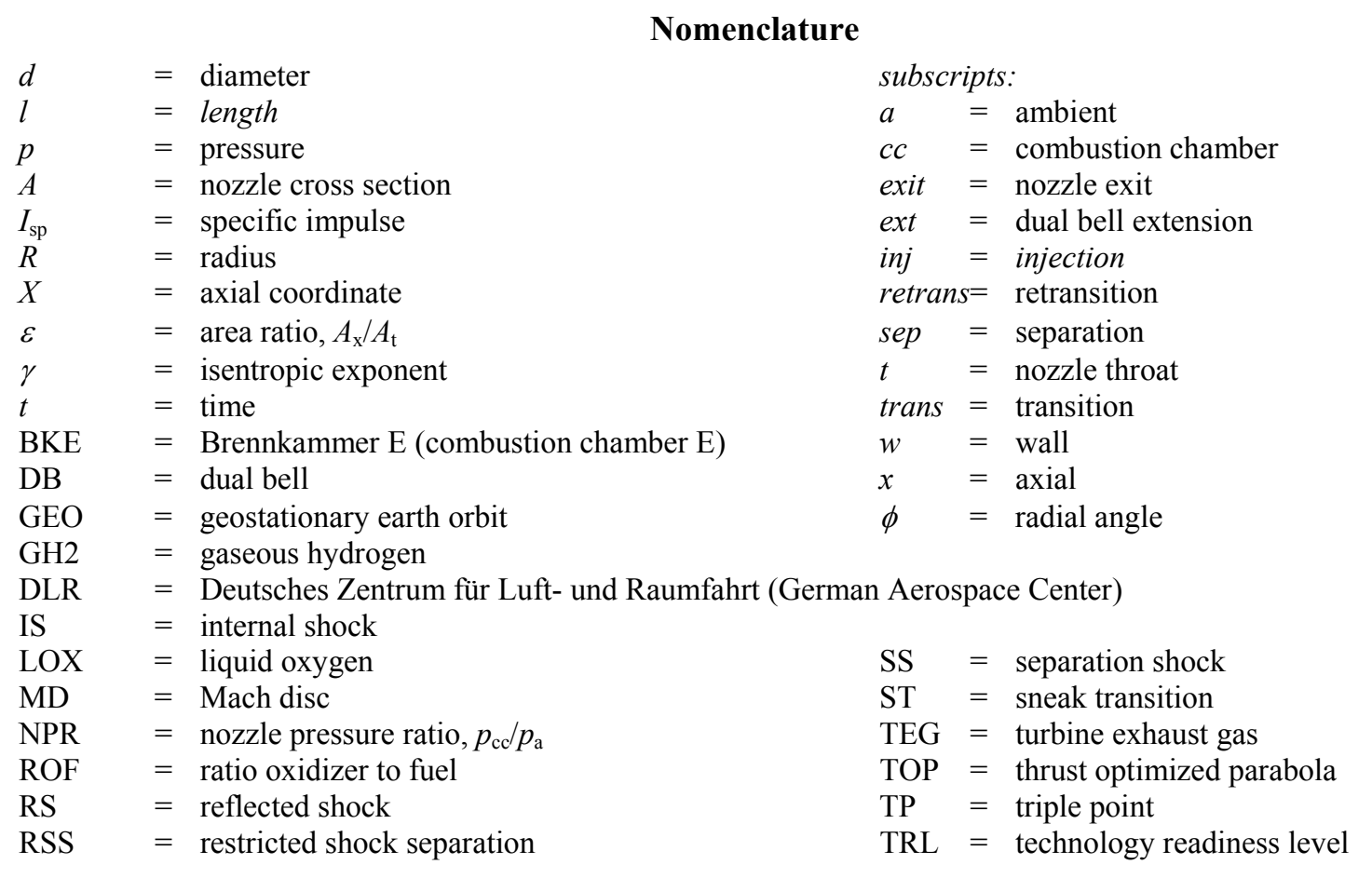

\section{Introduction}

Q urope's heavy lifter Ariane 5 features a parallel staged design. Its cryogenic main stage is supported by two - 1 solid boosters, generating the predominant part of the lift-off thrust. Ariane 5's original aim was the delivery of heavy payloads to a low earth orbit, like ATV to supply the space station or the abandoned shuttle Hermes. Nowadays, its dual GEO payload capability is in focus.

The main stage engine Vulcain 2 is ignited on ground to assure a proper running before the solid boosters are ignited and Ariane 5 takes off. Vulcain 2 passes during the rocket's ascent a wide range of ambient conditions, from sea level up to high vacuum. To prevent undesired flow separation on sea level, resulting in side loads that affect not only the engine but also the rocket structure and the payload itself, the nozzle area ratio was limited. However, an area ratio limitation results in overall performance losses. The engine's exhaust flow causes drag losses on sea-level

\footnotetext{
${ }^{1}$ Head of Nozzle Group, Institute of Space Propulsion, Langer Grund.

${ }^{2}$ Dr. Research Scientist, Institute of Space Propulsion, Langer Grund. 
as it is driven over expanded and expansion losses at high altitudes as it is here under expanded. To solve this conflict and to optimize the overall $I_{\text {sp }}$ of the engine during ascent the use of an altitude adaptive rocket nozzle, where the thrust generation is not only optimized on one specific altitude, comes into focus as the sub system with a promising performance gain. The commonly discussed nozzle concepts are plug nozzles, extendible nozzles and dual bell nozzles.
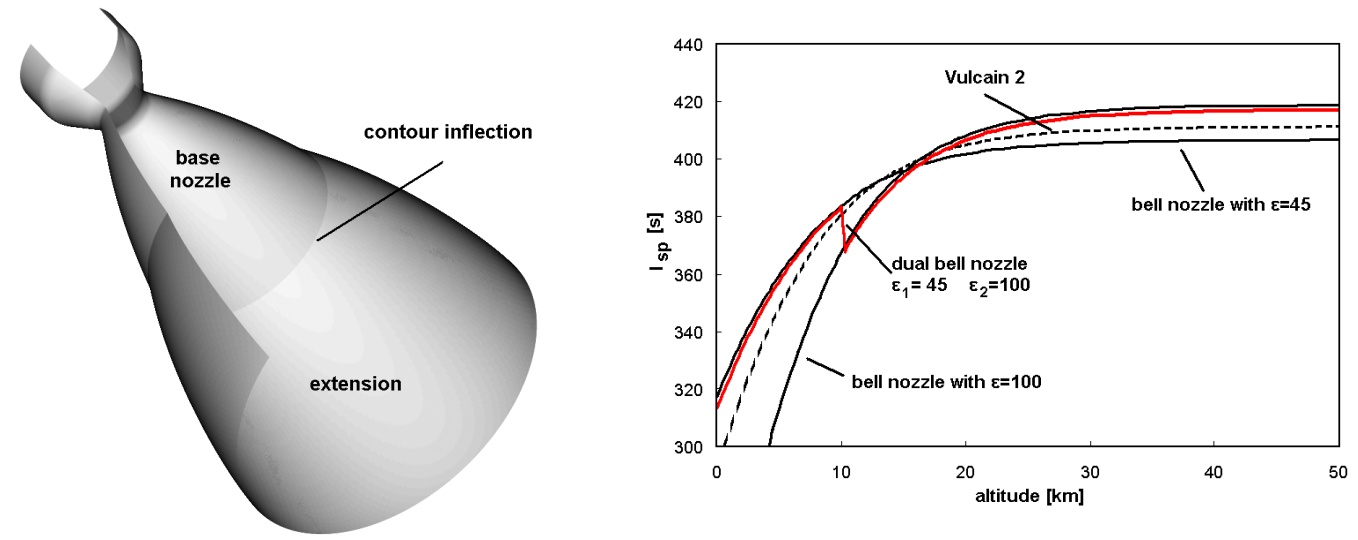

Figure 1. Dual bell nozzle. Sketch of dual bell nozzle (left), overall performance gain (right).
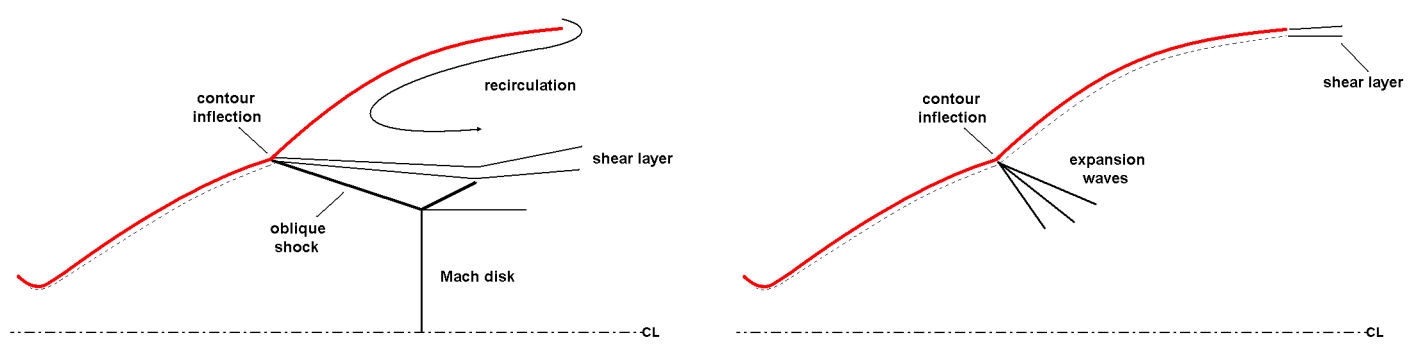

Figure 2. Dual bell's flow structure. During sea level (left) and high altitude mode operation (right).

The dual bell's major advantage is the absence of any moving parts. Only minor changes on the design and the structure of already operational rocket engines become necessary. Its characteristic wall contour inflection, dividing the nozzle into base and extension, offers a one-step altitude adaptation (Fig. 1, left). On sea level, the contour inflection forces the flow to separate controlled and symmetrically (Fig. 2, left). The base nozzle is full flowing and the extension is separated. This is called the sea level mode. Due to a smaller effective area ratio the sea level $I_{\mathrm{sp}}$ increases compared to a conventional nozzle (Fig. 1, right). At the designed altitude the flow attaches abruptly to the wall of the extension down to the exit plane (Fig. 2, right), resulting for a short time $I_{\text {sp }}$ loss but later on in a higher vacuum performance. The process is addressed as dual bell transition to high altitude mode.

Especially Vulcain 2 is a candidate for a dual bell application as its supersonic nozzle part is divided into a conventional, hydrogen cooled upper part and a lower part, being film cooled by the injected turbine pump exhaust gases. The film cooled metallic nozzle structure can be easily replaced by a dual bell extension. In this case special attention has to be paid on the cooling film, its expansion around the contour inflection, its stability inside the dual bell extension and its influence on the operation mode transition behaviour.

The German Aerospace Center (DLR) performed a lot of experimental studies on cold and warm flow subscale dual bell models to study the dual bell fundamentals. Tests were conducted on the transition, retransition and hysteresis behaviour $\left(G_{\text {énin }}{ }^{1}\right)$. The influence of the extension geometry and length, the inflection design, the side load behaviour and transition time were studied experimentally $\left(G^{2} \operatorname{nin}^{2}\right)$. A transition prediction could be developed and an intermediate flow state, addressed as Sneak Transition, was verified. The next steps are hot flow demonstrations and, especially with respect to Vulcain 2, the study of film cooling effects. The here presented hot flow subscale tests follow the logic of TRL level enhancement. Discussed are first results of the initial test campaign. 


\section{Experimental setup}

\section{A. Test facility}

The test were conducted at DLR's research and technology test facility P8 in Lampoldshausen, Germany. The facility is designed to study chemical and physical processes in high pressure combustion chambers. The facility consists of two test cells where all media being necessary for the study of cryogenic engines are provided. Liquid hydrogen as well as liquid oxygen are available for test specimens with interface pressures up to 360 bar. To assure realistic injector head conditions (most of the tested combustion chambers are water cooled for cost effectiveness) gaseous hydrogen can be cooled down to $100 \mathrm{~K}$ using a liquid nitrogen cooler.

The engines are mounted on a horizontal rig, enabling an access for optical measurement devices. All feeding lines are driven in blow down mode, meaning all fuel tanks are loaded with the needed feeding pressure. With high precision regulation valves the fuel mass flows can be adjusted with a fault less than $1 \%$. The test facility is designed to work under maximum load conditions for $15 \mathrm{~s}$. If the combustion chamber pressure and the mass flows are reduced a test duration of several minutes can be achieved.

\section{B. Test specimen}

The tests were performed using DLR's research thrust chamber BKE. Its chamber diameter is $d_{\mathrm{cc}}=50 \mathrm{~mm}$ and its throat diameter $d_{\mathrm{t}}=33 \mathrm{~mm}$. BKE features a LOX/GH2 injector head using coaxial injector elements, a water cooled cylindric copper combustion chamber (lenghth $l_{\mathrm{cc}}=250 \mathrm{~mm}$ ) and a likewise water cooled copper throat element forming the convergent-divergent nozzle contour up to a supersonic area ratio of $\varepsilon=5$. Where $\varepsilon$ is the ratio of any axial cross section $A_{\mathrm{x}}$ normalized by the nozzle throat cross section $A_{\mathrm{t}}$
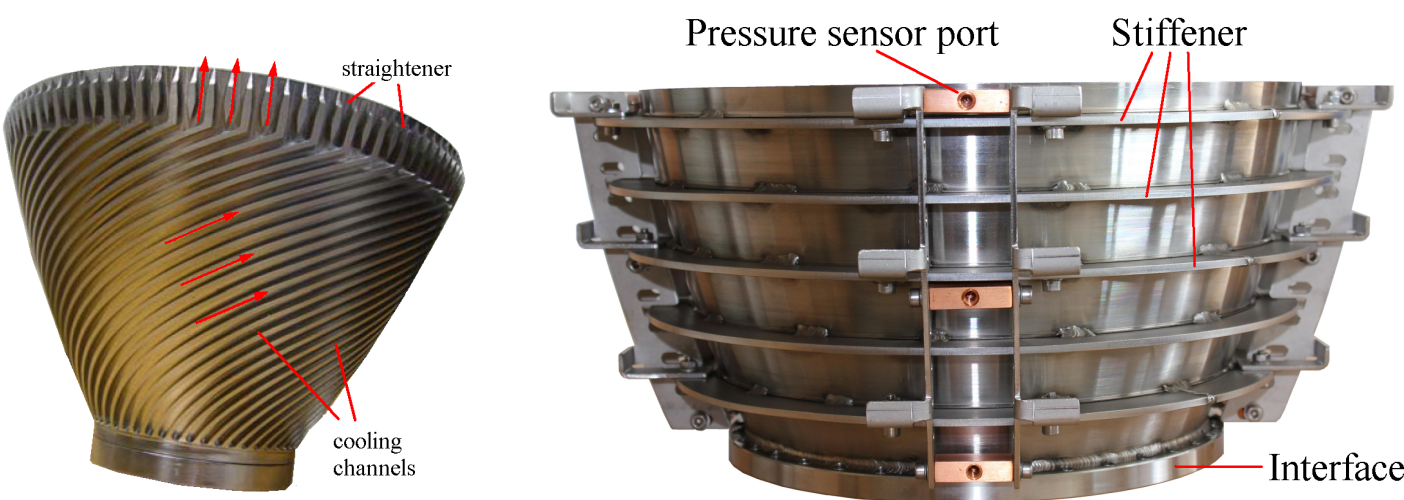

Figure 3. Supersonic nozzle. Base blank after milling (left, taken from Suslov et al. ${ }^{3}$ ), dual bell extension (right).

The supersonic nozzle, starting at the end of the thrust chamber $(\varepsilon=5)$ is divided in an upper (base) and a lower part. Its thrust optimized parabola (TOP) contour is Vulcain 2 like with a scaling rate of approximately 1:8.3. The base is made of Inconel 600 and convectively cooled with gaseous hydrogen, with an inlet temperature at ambient condition. The cooling channels are milled helically in the nozzle structure and finally closed by electroplating. Before entering the lower nozzle part as a cooling film the now heated hydrogen is turned by a straightener (Fig. 3, left), accelerated by a convergent-divergent throat section to supersonic velocity and injected parallel to the nozzle wall at $\varepsilon=32$ (see Fig. 4). More details on thrust chamber BKE and the base part can be found in Suslov et al. ${ }^{3}$.

The lower part, namely the dual bell extension, is also made of Inconel 600. Its wall thickness is $3 \mathrm{~mm}$ and the structure is enforced with 5 stiffeners (Fig. 3, right). An interface connects the extension and the base directly downstream the film injection. Retaining plates in all 4 quadrants support copper blocks used as pressure and hot wall temperature sensor ports. The extension is designed as a constant pressure contour, meaning the wall pressure profile has a zero gradient. The film was considered to be of constant wall pressure and density downstream the contour inflection and therefore a constant surface was added to each contour cross section to obtain the film offset. Details of BKE and the nozzle are given in table 1. 


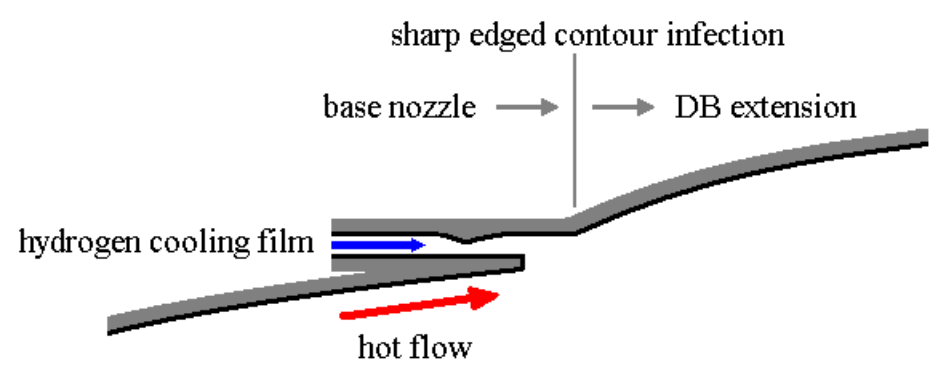

Figure 4. Film injection.

Table 1. Test specimen details.

\begin{tabular}{l|l|l} 
Combustion chamber diameter & $d_{\mathrm{cc}}$ & $50 \mathrm{~mm}$ \\
\hline Length of cylindrical chamber part & $l_{\mathrm{cc}}$ & $250 \mathrm{~mm}$ \\
\hline Throat diameter & $d_{\mathrm{t}}$ & $33 \mathrm{~mm}$ \\
\hline End area ratio of BKE & $\varepsilon_{\text {BKEend }}$ & 5 \\
\hline Area ratio of film injection & $\varepsilon_{\text {inj }}$ & 32 \\
\hline Exit area ration dual bell extension & $\varepsilon_{\text {exit }}$ & 77.42 \\
\hline Length of dual bell extension & $l_{\text {DBext }} / R_{\mathrm{t}}$ & 9.5
\end{tabular}

\section{Measurement techniques}

The dual bell extension was equipped with 17 pressure sensor ports in 4 quadrants carried out as copper blocks, where dynamic pressure sensors of Kulite XTEL-190M type could be directly mounted in. The blocks are connected via short thin metal tubes to the nozzle structure. The tubes were welded into the nozzle wall and feature an inner diameter of $0.9 \mathrm{~mm}$. After welding the inner nozzle side was finished by milling. The connection of tubes and blocks was carried out by shrinking. Additionally, the combination of blocks and inner diameter of the tubes allows to flush mount sealed Type-K thermo elements to measure the hot gas wall temperature. Nine Type-K thermo elements were applied on the outer nozzle surface (Fig. 5).

The setup was completed by a thermographic infrared system, imaging the outer nozzle side, and a side load reaction measurement. To measure the side load reaction pairs of strain gauges, connected as full Wheatstone bridges, were applied onto the metallic rods that connect the engine and the thrust frame. Infra red, side load reaction and wall temperature results are not part of the presented discussion.

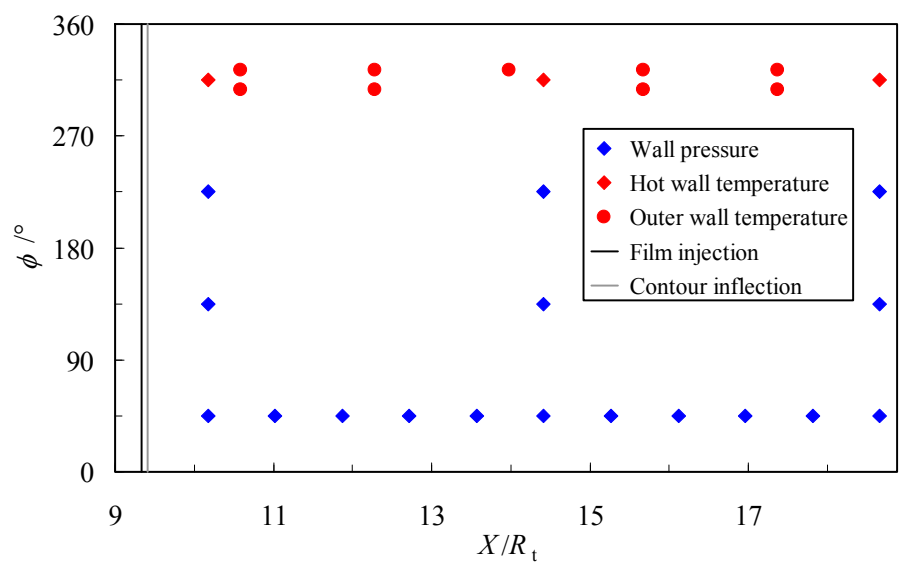

Figure 5. Measurement application. 


\section{Test sequence}

Figure 6 gives the test sequence with 3 subsequent up and down rampings to a maximum combustion chamber pressure of $p_{\mathrm{cc}}=115 \mathrm{bar}$. The ramp gradients are +/- 15,10 and $5 \mathrm{bar} / \mathrm{s}$, respectively. With these different gradients the slow ambient pressure decrease during ascent of the rocket as well as a rapid combustion chamber pressure change should be simulated. A combustion chamber pressure decrease with a delayed switchback to the nominal value might become necessary during ascent of a rocket to avoid a fluctuating dual bell mode transition within the buffeting phase, caused by huge after body pressure fluctuations and resulting in undesired side loads.

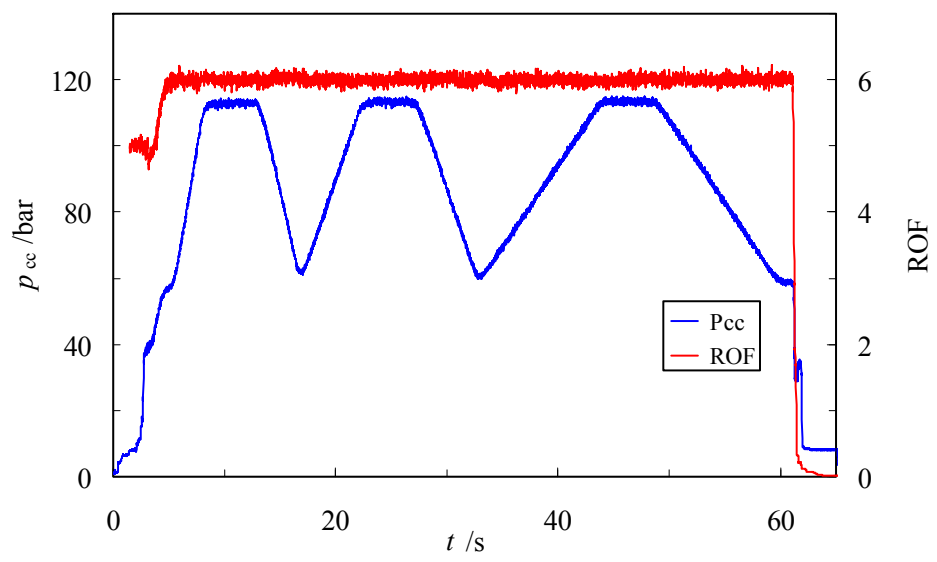

Figure 6. Test sequence.

The engine was driven with a start up ROF of 5 passing to a value of 6 for the remaining test sequence. This procedure follows the standard executed by Suslov et al. ${ }^{3}$ with his complete Vulcain 2 like nozzle. The test runs differ in the cooling film mass flow rate which were $0.175,0.2$ and $0.22 \mathrm{~kg} / \mathrm{s}$, respectively. Table 2 gives an overview of the film to hot gas mass flow ratios.

Table 2. Ratio film to hot gas mass flow.

\begin{tabular}{l|c|c} 
& Max. NPR & Mass flow ratio \\
\hline Run 3 & 100.3 & 0.0480 \\
\hline Run 4 & 115.6 & 0.0417 \\
\hline Run 5 & 115.4 & 0.0418 \\
\hline Run 6 & 115.3 & 0.0473 \\
\hline Run 7 & 115.7 & 0.0525 \\
\hline Vulcain 2 & 117.0 & $0.0416^{*}$ \\
& & $*$ TEG plus dump mass flow
\end{tabular}

Due to test stops provoked by thrust chamber redlines and the lack of facility occupation time, only five successful test could be run within this initial test campaign.

\section{Experimental results and discussion}

\section{A. Reattached flow condition}

During start up and shut down a flow state called restricted shock separation (RSS) occurred. It can be easily detected by interpreting the wall pressure profile (Fig. 7). The over expanded wall pressure with values below ambient pressure is followed by a section with a positive wall pressure gradient, up to a peak value above ambient pressure and a subsequent expansion. The peak value is caused by the reattachment shock.

RSS is typical for TOP nozzles were a internal shock (IS) is generated shortly downstream the nozzle throat (see Fig.9, left). The transition within the wall contour slope from the convex throat radius to the concave parabola initiates compression waves that merge to a weak shock. The shock continues through the flow field and hits the strong normal shock or Mach disc (MD) and is redirected as reflected shock (RS) towards the nozzle wall. Internal shock, reflected shock and Mach disc form the triple point (TP). The reflected shock interacts and crosses the oblique separation shock (SS) coming from the wall, where the boundary layer lifts off and turns into the shear 
layer. The oblique separation shock redirects a part of the flow towards the center axis and the reflected shock another part towards the nozzle wall. The impulse balance of both redirected flows decides whether the total flow is directed parallel to the center axis and forms out the exhaust jet or is redirected towards the nozzle wall and reattaches.

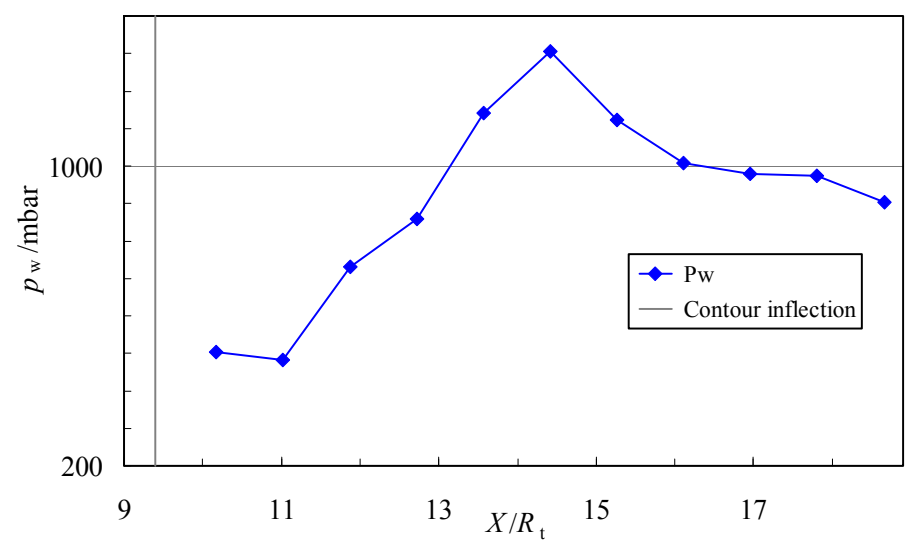

Figure 7. Wall pressure profile in dual bell extension during RSS condition.

In opposite to Vulcain, Vulcain2 doesn't show a RSS condition. The reason for this is that the moving position of the flow separation on the nozzle wall during start up of the engine is for a short period fixed at the edge of the TEG injection slots and therefore the impulse downstream of the oblique separation shock always dominates. With this background it was surprising that RSS occurred, specially as the nozzle contour with its film injection position was Vulcain 2 like.
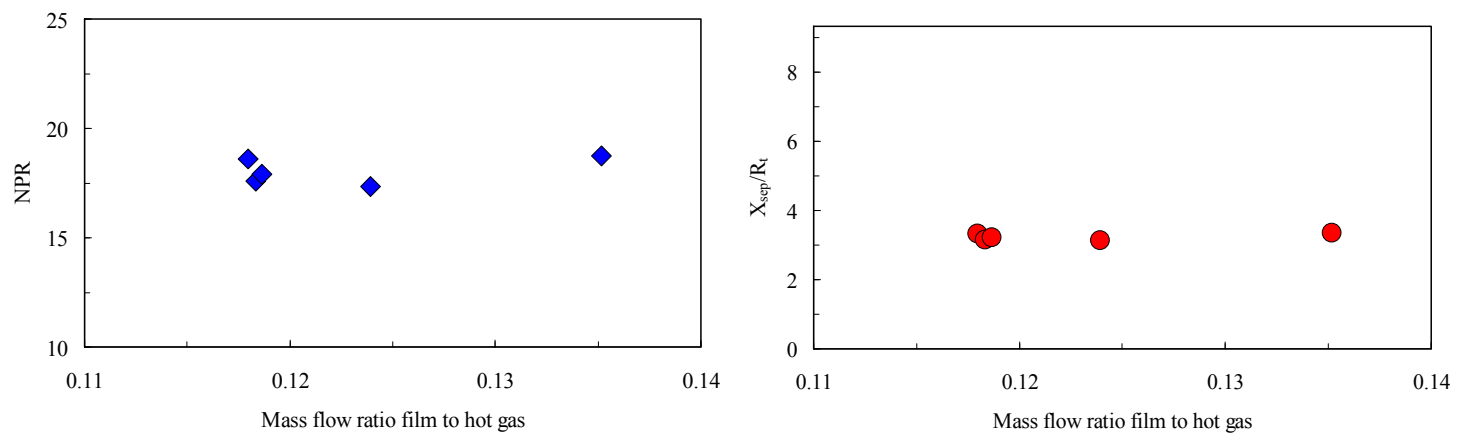

Figure 8. Influence of film to hot gas ratio. On start of RSS( left) and separation position (right).

The first difference between subscale engine BKE and Vulcain 2 is the constant film mass flow rate while ramping up and down the combustion chamber pressure with its related hot gas mass flow, in opposite to Vulcain 2 where hot gas and film (TEG) mass flow are coupled via the turbine performances. If the flow separation inside the base nozzle depends on the suction capacity of the film injection a higher film mass flow should shift the separation downstream and RSS flow state should subsequently start at lower nozzle pressure ratios $\left(\mathrm{NPR}=p_{\text {cc }} / p_{\mathrm{a}}\right)$. Figure 8 , left gives the NPRs of the transitions to RSS as a function of the tested film to hot gas mass flow ratios and Fig. 8, right gives the depending separation positions inside the base nozzle, where the position was calculated using the separation criteria $p_{\text {sep }} / p_{\mathrm{a}}=1 / \mathrm{Ma}_{\text {sep }}\left(\operatorname{Stark}^{4}\right)$ and the wall Mach numbers were calculated using TDK94 with a ROF of 5. It can be seen that no deciding influence of the film mass flow neither on the transition to RSS nor on the separation position is given. 

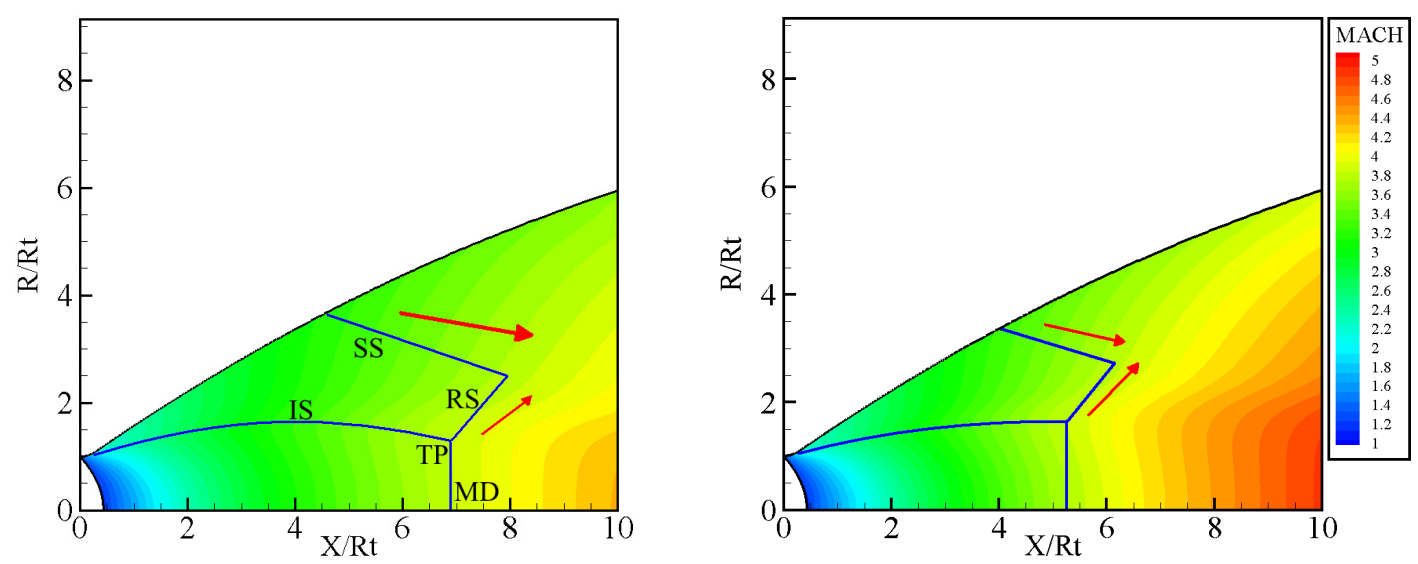

Figure 9. Flow fields with overlaid shock structure sketches. ROF 7.2 left and ROF 5 right.

The second difference is the start up ROF of 5 compared to 7.2, where Vulcain 2 nozzle was designed for (see Ref. 5). The flow field inside the nozzle mainly depends on the gas properties, e.g. the isentropic exponent $\gamma$. For a given nozzle contour a lower ROF (higher $\gamma$ ) leads to higher local Mach numbers. An example is given in Fig. 9 where the flow field inside the base nozzle is shown for the design ROF of 7.2 (left) and BKE's start up ROF of 5 (right) using TDK94 calculations. Both flow fields were overlaid with a sketch of the shock system, illustrating the shock positions for an equal separation NPR. For better readability boundary and shear layers as well as slip lines, the shocks and expansion waves downstream the crossing of oblique separation and reflected shock are omitted. It clearly can be seen that both, the origin of the oblique separation shock at the nozzle wall and the triple point are shifted upstream for ROF of 5. But, the triple point is much more shifted upstream. This leads to an impulse balance downstream the reflected and the separation shock that promotes the flow to be redirected to the nozzle wall and to reattach. So, the main reason for RSS appearance during the presented test campaign was the start up ROF of 5.

\section{B. Sneak Transition}

The sneak transition is an intermediate flow state between sea level and high altitude operation mode and it precedes the abrupt actual transition (see Génin ${ }^{1}$ ). In theory the Prandtl-Mayer expansion fan, located at the sharp edged contour inflection, leads to a steep dip in the wall pressure profile down to the constant pressure value of the dual bell extension (Fig. 10). In reality, due to boundary layer viscosity effects, down stream the contour inflection a so called inflection region is given, where a strong negative wall pressure gradient is present. The flow separation that is fixed for a given NPR interval at the contour inflection starts from a certain NPR value to move in this region.

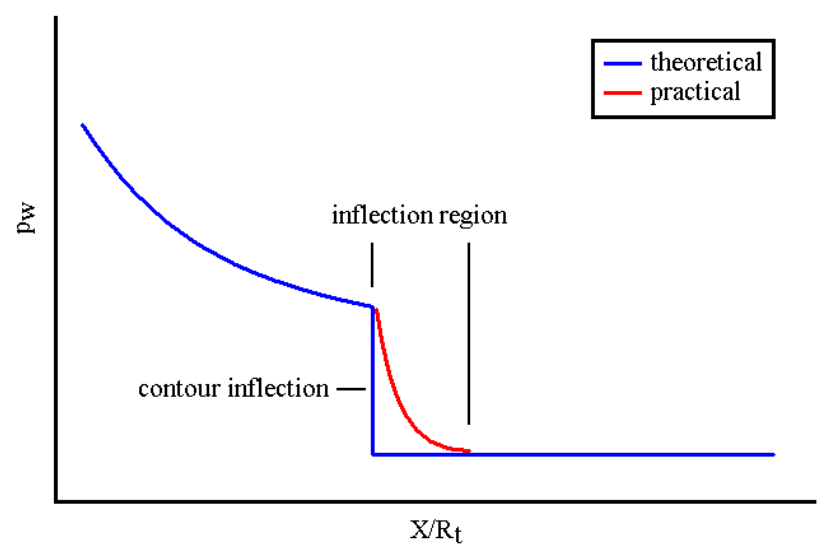

Figure 10. Sketch of wall pressure profile within inflection region. 
This is the start of the sneak transition. Here, the flow behaves like a normal separation, leading e.g. to side loads being comparable to usual separated nozzle flows $\left(G^{\prime} \operatorname{nin}^{7}\right)$. Once the flow separation reaches the end of the inflection region, the actual transition takes places and the flow jumps abrupt, in milliseconds, to the end of the dual bell extension as no stable separation position can be found. The typical length of the inflection region was found in cold as well as warm flow tests (without film injection) to be in the range of $1 R_{\mathrm{t}}$.
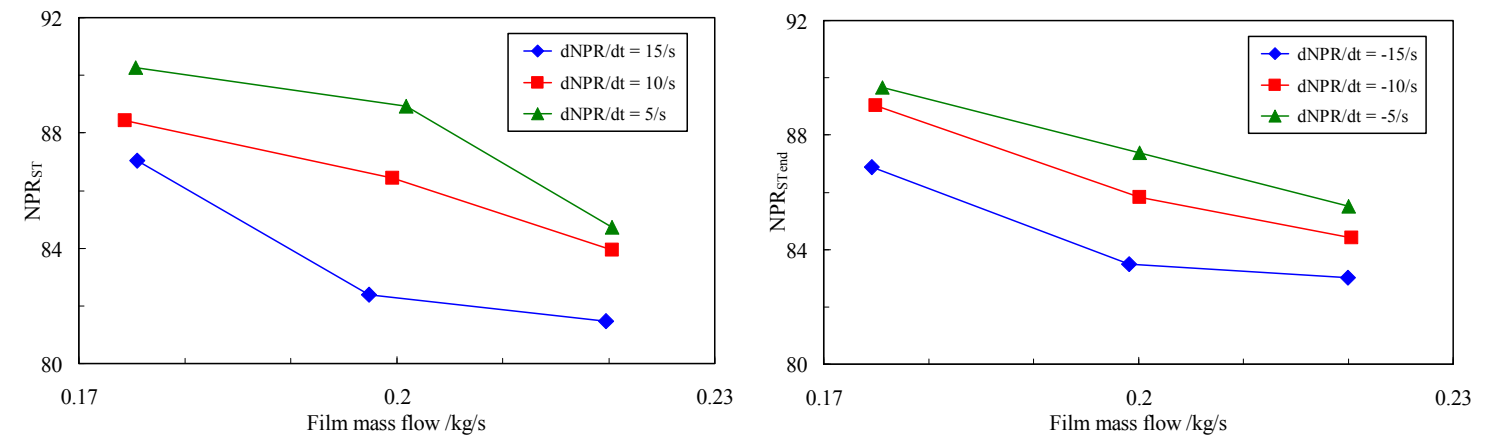

Figure 11. Sneak transition NPR as function of film mass flow. Up ramping left and down ramping right.

Figure 11 left gives the NPRs were sneak transitions started as a function of the film mass flow. As awaited an increased film mass flow led to a decreased start NPR. As the transonic film throat was not changed, the film mass flow increase led to increased film injection pressures, compared to the design point of $0.175 \mathrm{~kg} / \mathrm{s}$. Due to the interaction with the hot gas flow a recompression shock forms out and higher wall pressures cause a premature start of the sneak transition flow state. A second effect can be seen for the subsequent up and down rampings. The start NPRs are shifted to higher values. It is not the influence of the decreased combustion chamber pressure gradient but the before heated and therefore widened dual bell extension structure that increases the inflection angle and the dedicated expansion. Higher wall pressures, meaning a higher combustion chamber pressure was needed to push the flow separation into the inflection region.

Figure 11 right gives the NPRs where the sneak transition ends during ramping down. The values for different $p_{\mathrm{cc}}$ gradients are shifted slightly up, again owing to the heated structure.
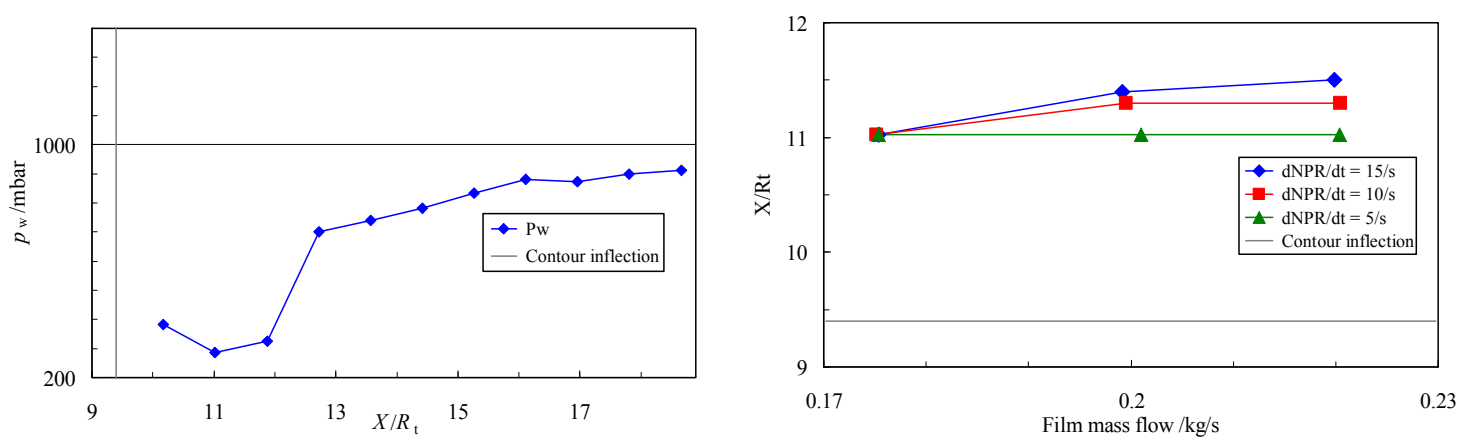

Figure 12. Separation position. Most down stream separation left and all achieved positions right.

Normally, a distinct phase of sneak transition could not be observed during down ramping of the combustion chamber pressure $p_{\mathrm{cc}}$ as the given hysteresis between transition and retransition NPR $\left(\mathrm{NPR}_{\mathrm{retrans}}<\mathrm{NPR}\right.$ trans $)$ and the abrupt jump of the flow back to the contour inflection during retransition prevents the detailed study or even the detection of this intermediate flow state. So, the presented results, studying the end of the sneak transition phase, gives a hint that no actual transition took place. Figure 12, left gives the wall pressures within the dual bell extension for a maximum $p_{\mathrm{cc}}$ of 115.5 bar and a film mass flow of $0.220 \mathrm{~kg} / \mathrm{s}$. It determines the most down stream separation position reached during the test campaign. The separation is still located within the inflection region and its negative wall pressure profile. The data indicate that the length of the inflection region is in minimum of two times the nozzle throat $R_{\mathrm{t}}$. Such an influence of the cooling film to elongate the inflection region was predicted by CFD studies of e.g. Martelli et al. ${ }^{8}$. The CFD results are not so much pronounced as found in the experiments. 
Martelli theoretically as well as Proshchanka ${ }^{9}$ experimentally showed that an increasing film mass flow leads at first to an increased transition NPR, up to a distinct maximum and a subsequent transition NPR reduction. The data presented in Fig. 11, right, where the maximal achieved separation positions are given for an equal NPR of around 115.5, illustrate that an increased film mass flow led to a downstream shift of the separation positions. This indicates, the conducted test variations were in the region of NPR reduction.

\section{Conclusion}

The initial test campaign using BKE and its Vulcain 2 like base nozzle yield first experiences in operating a film cooled subscale dual bell nozzle under representative conditions. The surprising reattached flow condition during engine start up was found to be a consequence of the chosen low start up ROF and will be avoided by changing the sequence in subsequent test campaigns. An actual transition to high altitude mode operation was not achieved but the obtained sneak transition results illustrate that a film mass flow increase will lower the transition NPR. Attention has to be paid to the thermal structure effects. A full scale demonstrator will be operated for a certain period of time within sea level operation mode before transition to high altitude mode is initiated, in order to simulate the rocket ascent. During this period the dual bell extension will heat up. The consequential deformation has to be taken into account during design process to assure a proper mode transition.

The inflection region was found to be much longer than awaited. This will effect the side load behavior during the intermediate flow of state sneak transition and has to be studied in detail.

\section{Acknowledgments}

The authors would like to thank Dr. Dmitry Suslov for setting up the test sequences, Philipp Gross for operating combustion chamber BKE and the P8 crew for good teamwork.

\section{References}

${ }^{1}$ Nürnbeger-Génin, C. and Stark, R., "Flow transition in dual bell nozzles", Shock Waves, Vol. 19, No. 3, 2009, pp. 265-270, DOI: $10.1007 / \mathrm{s} 00193-008-0176-4$.

${ }^{2}$ Nürnbeger-Génin, C. and Stark, R., "Experimental Study on Flow Transition in Dual Bell Nozzles", Journal of Propulsion and Power, Vol. 26, No. 3, 2010, pp. 497-502, DOI: 10.2514/1.47282.

${ }^{3}$ Suslov, D. I., Arnold, R. and Haidn, O.J., "Experimental Investigation of Cooling Film Efficiency in the Extension of a LOX/LH2 Subscale Combustion Chamber", Proceedings of the 4th European Conference for Aerospace Sciences (EUCASS) on Disc [CD-ROM] and online, ID 558, 4-7th July 2011, St Petersburg, Russia, 2011.

${ }^{4}$ Stark, R. and Wagner, B., "Experimental study of boundary layer separation in truncated ideal contour nozzles", Shock Waves, Vol. 19, No. 3, 2009, pp. 185-191, DOI: 10.1007/s00193-008-0174-6.

5EADS Astrium Space Transportation, "Vulcain 2 Thrust Chamber", Spec Sheet.

${ }^{6}$ Volvo Aero, "Vulcain 2 Nozzle", Spec Sheet.

${ }^{7}$ Génin, C. and Stark, R., "Side Loads in Sub-scale Dual Bell Nozzles", Journal of Propulsion and Power, Vol. 27, No. 4, 2011.

${ }^{8}$ Martelli, E., Scaramuzzino, F. and Nasuti, F., "Secondary Gas Injection Effect on Dual-Bell Nozzle Flow Transition", Proceedings of the 4th European Conference for Aerospace Sciences (EUCASS) on Disc [CD-ROM] and online, ID 219, 4-7th July 2011, St Petersburg, Russia, 2011.

${ }^{9}$ Proshchanka, D., Yonezawa, K. ,Koga, H., Tsukuda, H., Tsujimoto, Y., Kimura, T., Yokota, K. and Pasini, A., "Control of Operation Mode Transition in Dual-Bell Nozzle with Film Cooling", $46^{\text {th }}$ AIAA/ASME/SAE/ASEE Joint Propulsion Conference, AIAA 2010-6815, 25-28th July 2010, Nashville, TN, 2010. 